\title{
Photometry and polarimetry of the nucleus of comet 2P/Encke ${ }^{\star}$
}

\author{
H. Boehnhardt ${ }^{1}$, G. P. Tozzi ${ }^{2}$, S. Bagnulo ${ }^{3}$, K. Muinonen ${ }^{4}$, A. Nathues ${ }^{1}$, and L. Kolokolova ${ }^{5}$ \\ ${ }^{1}$ Max-Planck-Institute for Solar System Research, Max-Planck-Str. 2, 37191 Katlenburg-Lindau, Germany \\ e-mail: boehnhardt@mps.mpg.de \\ 2 INAF Arcetri Observatory, Florence, Italy \\ Armagh Observatory, Armagh, Northern Ireland, UK \\ 4 University Observatory, Helsinki, Finland \\ ${ }^{5}$ University of Maryland, College Park, Maryland, USA
}

Received 7 April 2008 / Accepted 27 June 2008

\section{ABSTRACT}

Context. Comet 2P/Encke, a short-period comet, is a favorable target for studies of light scattering by bare cometary nuclei. These studies enable assessment of the nucleus size, the material albedo, and the light-scattering properties of the cometary surface. Observations of 2P/Encke were completed between October to December 2006 when the comet approached the Sun between 2.7 and $2.1 \mathrm{AU}$.

Aims. We characterize the activity of the cometary coma and the physical properties of the nucleus of $2 \mathrm{P} /$ Encke such as its size, albedo, colors, spectral slope, surface roughness, porosity, and single-particle properties.

Methods. Broadband imaging photometry and broadband and narrowband linear polarimetry is measured for the nucleus of $2 \mathrm{P} /$ Encke over the phase-angle range $4-28 \mathrm{deg}$.

Results. An analysis of the point spread function of the comet in polarimetric images reveal only weak coma activity in $2 \mathrm{P} /$ Encke, corresponding to dust production of the order of $0.05 \mathrm{~kg} / \mathrm{s}$. Over the measured phase-angle range the nucleus displays a color independent photometric phase function of almost linear slope $(\beta=0.050 \pm 0.004 \mathrm{mag} / \mathrm{deg})$. The absolute $R$ filter magnitude at zero phase angle is $15.05 \pm 0.05 \mathrm{mag}$ and corresponds to an equivalent radius for the nucleus of $2.43 \pm 0.06 \mathrm{~km}$ (adopted albedo of 0.047 ). The nucleus color $V-R$ was measured to be $0.47 \pm 0.07$, suggesting a spectral slope $S^{\prime}$ of $11 \pm 8 \% / 100 \mathrm{~nm}$. The phase function of linear polarimetry in the $V$ and $R$ filter shows a widely color independent linear increase with phase angle $(0.12 \pm 0.02 \% / \mathrm{deg})$. The test of the empirical albedo-polarization relationship for asteroids reveals unreasonably high albedo values for the nucleus. We find discrepancies in the photometric and polarimetric parameters between 2P/Encke and other minor bodies in the solar system, which may indicate significant differences in the surface material properties and light-scattering behavior of the bodies.

Conclusions. The linear polarimetric phase function of $2 \mathrm{P} /$ Encke presented here is the first ever measured for a cometary nucleus. It provides encouragement to future studies of cometary nuclei in order to characterize the light-scattering behavior of comets on firm empirical grounds and provide suitable input to a comprehensive modeling of the light scattering by cometary surfaces.

Key words. comets: general - comets: individual: 2P/Encke - minor planets, asteroids - Kuiper Belt

\section{Introduction}

After its discovery in 1786 , the short-period comet $2 \mathrm{P} /$ Encke was observed during almost 60 perihelion passages and is one of the most significantly (more than 40 papers in refereed journals) and longest (over 200 years) studied comets. With an orbital revolution period of about 3.3 years, it is closer to the Sun than typical Jupiter family comets (JFC). Levison et al. (2006) suggested that $2 \mathrm{P} /$ Encke may be a rare example of a comet in an orbit detached from Jupiter's immediate control (like JFCs are) due to gravitational interaction with the terrestrial planets. They also argued that the nucleus may have been in a quiescent state for a long part of the transition period into its current orbit.

A series of scientific papers dealt with the nucleus properties of 2P/Encke. Fernandez et al. (2000) measured an effective nuclear radius of $2.4 \pm 0.3 \mathrm{~km}$, an axial ratio of at least 2.6 , a geometric albedo of $0.047 \pm 0.023$, which is in the typical range for comets, and a rather steep photometric phase function with a linear slope of $0.06 \mathrm{mag} / \mathrm{deg}$ which was later confirmed by Jewitt (2002). A wider range of values for the effective radius

* Based on observations performed under program 078.C-0509 at the European Southern Observatory, Cerro Paranal, Chile.
(2.4-3.7 km) was determined from radar echoes of the comet (Harmon \& Nolan 2005). Colors of $V-R=0.39 \pm 0.06$ and $B-V=0.73 \pm 0.06$ (Lowry \& Weissman 2007) indicated only moderate spectral slope $S^{\prime}$ of the nuclear surface as found by Jewitt (2002) to be $8.9 \pm 1.6 \% / 100 \mathrm{~nm}$. The rotation period was a matter of debate in various papers without a final conclusion (see Belton et al. 2005, and references therein). Attempts to determine the orientation of the rotation axis were made by Sekanina (1991) and Festou \& Barale (2000). Jewitt (2004) measured a slightly positive linear polarization of the nucleus at a single phase angle (polarization of $1.0-1.9 \pm 0.5 \%$ at $22^{\circ}$ ) in the visible. The activity profile of the nucleus appears to be unusual with a coma being visible around aphelion (Fernandez et al. 2005), but no activity during its inbound arc as close as 1.4 AU solar distance (Jewitt 2004).

The likely absence of activity combined with the predicted nucleus brightness enabled us to attempt successfully (i.e. with sufficient signal-to-noise and without significant coma contamination) polarization measurements of $2 \mathrm{P} /$ Encke. We report below on the results of the phase-angle-resolved linear polarimetry and quasi-simultaneous broadband photometry of this cometary nucleus in the visible wavelength range. The main aim of 
these measurements is to constrain the light-scattering properties of the nuclear surface, i.e. the single-scattering albedo and mean free path as a proxy for the typical grain size to be obtained through modeling. Furthermore, we could test the albedo-polarization relationship known from asteroids (Zellner \& Gradie 1976; Zellner et al. 1977; Lupishko \& Mohamed 1996; Cellino et al. 1999), for a cometary nucleus. Our results are the first ever linear polarimetric observations of a cometary nucleus over a wider phase-angle range.

\section{Observations and data reduction}

The observations of comet $2 \mathrm{P} /$ Encke were performed at Unit Telescope 2 of the Very Large Telescope observatory (VLT) at Cerro Paranal in Chile. The FORS1 instrument ${ }^{1}$ was used in imaging and polarimetric modes with broadband Bessell $V$ and $R$ and two narrowband filters of central wavelengths and widths $485 \mathrm{~nm} / 37 \mathrm{~nm}$ and $834 \mathrm{~nm} / 48 \mathrm{~nm}$, respectively; the filters are abbreviated below as " $b_{\text {cont }}$ " for the blue and " $r_{\text {cont }}$ " for the red one. The narrowband filters were selected from the available set of FORS filters such that their transmission ranges contained little to no contamination from emission bands of possible coma gases, i.e. the narrowband filters cover mostly surface and dust reflected sunlight. The $b_{\text {cont }}$ and $r_{\text {cont }}$ filters were only used during the polarimetric measurements at the beginning of the program, since, due to the absence of significant coma contamination, we decided to maximize the signal-tonoise level by exclusive usage of the broadband $V$ and $R$ filters from 28 Oct. 2006 onwards (see Table 1).

\subsection{Observations}

Service mode observations were the most appropriate choice given the specific requirements of this program: the short duration $(1-1.5 \mathrm{~h})$ of individual observing runs; a frequent, but irregular run schedule to cover the orbit of $2 \mathrm{P} /$ Encke at about regular phase-angle differences between 4 and 30 deg phase angle; and acceptable sky conditions for the observations. To the advantage of a dense phase-angle coverage, we relaxed the requirements for seeing $\left(2^{\prime \prime}\right)$ and sky transparency (thin cirrus or better; in most cases the measurements were performed with clear sky) for our program. The comet was positioned at the center of the instrument field of view, a location that placed it automatically in the center of the central strip of the FORS1 polarimetric mask used to separate the two beams of polarized light produced by the Wollaston prism in polarimetric mode. Exposure series of linear polarimetry of the comet were taken for each filter at $\lambda / 2$ retarder plate settings of position angles (with respect to North Celestial Meridian) $0^{\circ}, 22.5^{\circ}, 45^{\circ}, 67.5^{\circ}, 90^{\circ}, 112.5^{\circ}$, $135^{\circ}$, and $157.5^{\circ}$. The filter imaging and polarimetric exposure series were obtained subsequently within a few minutes and less than $1 \mathrm{~h}$, respectively, per observing run. Differential autoguiding on a nearby star at the speed of the comet in the sky was applied during the observations and the usual calibration exposures according to the FORS instrument calibration plan were taken (i.e. for imaging: photometric standard star field, bias and sky flatfield exposures; for the polarimetry: bias and screen flatfield exposures plus a polarized and unpolarized standard star). The observing program was executed in 9 runs during 1 Oct. and 14 Dec. 2006. Details of the observations are summarized in Table 1.

\footnotetext{
1 See

http://www. eso.org/instruments/fortechnicaldetails
}

\subsection{Reduction of the photometry data}

All images were bias subtracted, and images used for photometry were then divided by a master flat field obtained from four sky flats taken at twilight. For the background subtraction, we subtracted in a first step a constant value that was estimated in regions far from the comet photometric center, where the contribution of a possible coma was negligible. A further correction was evaluated and subtracted by measuring the $\Sigma A f$ function, defined by Tozzi et al. (2004), versus the projected nucleocentric distance, $\rho$. The $\Sigma A f$ function is defined by the product of the geometric albedo multiplied with the total area covered by the solid component (usually the cometary dust; A'Hearn et al. 1995) in an annulus of radius $\rho$ and unitary depth (Tozzi et al. 2004). For a normal comet, the $\Sigma A f$ function is constant, and in the case of no coma it should be zero. Following a trial and error procedure, different background values were subtracted until the $\Sigma A f$ function was measured to be constant versus $\rho$. As shown in Fig. 2, $\Sigma A f$ shows a peak at the cometary center, where the light-scattering contribution from the nucleus dominates, and decreases to values close to zero at larger projected distances from the nucleus (for a more detailed discussion see below). Finally, the images were calibrated both in magnitude and in $A f$. The value $A f$ describes the product of the mean albedo $\mathrm{A}$ and the filling factor $f$ of the dust grains in a measurement aperture of radius $\rho$. The product $A f \rho$ is a measure of the dust production of the comet (for details on $A f \rho$ see A'Hearn et al. 1995). Using standard star images we determined photometric zeropoints for the respective observing nights assuming atmospheric extinction coefficients of 0.114 and $0.065 \mathrm{mag} / \mathrm{airmass}$ and instrument color coefficients of 0.03 and 0.06 (plus solar colors for the comet to a first approximation) for $V$ and $R$ filters, respectively. The final two photometric parameters could not be determined from the available calibration images directly and were adopted after a careful analysis of the respective information on ESO's data quality information web page for the FORS instrument ${ }^{2}$. The cometary photometry was measured using a constant aperture diameter of $6^{\prime \prime}$.

\subsection{Reduction of the linear polarimetry data}

All polarimetric images were bias subtracted and flatfield corrected. For the background subtraction, we followed the same strategy used for the imaging data of the comet, by treating the ordinary and extra-ordinary beams separately. A further refinement in the background estimate was obtained by measuring (for each position angle of the retarder waveplate) the ratio

$h=\frac{\left(f^{\mathrm{o}}-f^{\mathrm{e}}\right)}{\left(f^{\mathrm{o}}+f^{\mathrm{e}}\right)}$,

where $f^{\circ}$ is the flux in the ordinary beam and $f^{\mathrm{e}}$ the flux in the extraordinary beam, in annuli of increasing radius. We then readjusted the value used for background subtraction until the measured $h$ values became independent of the projected nucleocentric distance $\rho$. In the $R$ band, typically, the background values obtained after the first step (i.e. estimated from regions far from the cometary nucleus) were approximately $800 \mathrm{e}^{-}$; the first correction, based on the analysis of the $\Sigma A f$ function, was approximately $10-20 \mathrm{e}^{-}$; the final correction derived by insisting that the ratio $h$ was constant with $\rho$, was approximately $1-2 \mathrm{e}^{-}$.

\footnotetext{
${ }^{2}$ See http://www.eso.org/observing/dfo/quality
} 
Table 1. Observing log and measurement results of the photometry and linear polarimetry of comet 2P/Encke.

\begin{tabular}{|c|c|c|c|c|c|c|c|c|c|c|}
\hline $\begin{array}{c}\text { EPOCH } \\
\text { (UT) }\end{array}$ & $\begin{array}{c}\text { PHASE } \\
\left({ }^{\circ}\right)\end{array}$ & $\begin{array}{c}r \\
(\mathrm{AU})\end{array}$ & $\begin{array}{c}\Delta \\
(\mathrm{AU})\end{array}$ & Filter & $\begin{array}{c}H_{0} \\
(\mathrm{mag})\end{array}$ & $\begin{array}{l}P_{Q} \\
(\%)\end{array}$ & $\begin{array}{l}P_{U} \\
(\%)\end{array}$ & $\begin{array}{c}P_{\mathrm{L}} \\
(\%)\end{array}$ & $\begin{array}{l}\zeta \\
\left({ }^{\circ}\right)\end{array}$ & Notes \\
\hline 2006-10-01 01:40:02 & 4.01 & 2.75 & 1.76 & $\bar{V}$ & & $-0.90 \pm 0.51$ & $0.03 \pm 0.51$ & 0.90 & $89 \pm 17$ & \\
\hline 2006-10-02 01:48:31 & 4.01 & 2.74 & 1.75 & $\mathrm{~V}$ & & $-0.70 \pm 0.58$ & $-1.20 \pm 0.59$ & 1.39 & $120 \pm 17$ & \\
\hline 2006-10-02 02:04:20 & 4.02 & 2.74 & 1.75 & $b_{\text {cont }}$ & & $-0.78 \pm 0.42$ & $-0.42 \pm 0.43$ & 0.89 & $104 \pm 19$ & \\
\hline 2006-10-02 02:20:12 & 4.02 & 2.74 & 1.75 & $R$ & & $-0.68 \pm 0.32$ & $-0.07 \pm 0.32$ & 0.68 & $93 \pm 15$ & \\
\hline 2006-10-02 02:40:03 & 4.02 & 2.74 & 1.75 & $r_{\text {cont }}$ & & $-1.45 \pm 0.40$ & $-1.24 \pm 0.40$ & 1.91 & $110 \pm 9$ & \\
\hline 2006-10-02 02:56:20 & 4.03 & 2.74 & 1.75 & $R$ & $15.16 \pm 0.07$ & & & & & \\
\hline 2006-10-02 02:57:55 & 4.03 & 2.74 & 1.75 & V & $15.66 \pm 0.07$ & & & & & \\
\hline 2006-10-12 03:47:04 & 8.09 & 2.66 & 1.71 & $V$ & & $-0.28 \pm 0.36$ & $-0.20 \pm 0.35$ & 0.35 & $107 \pm 41$ & \\
\hline 2006-10-12 04:03:19 & 8.10 & 2.66 & 1.71 & $b_{\text {cont }}$ & & $-0.50 \pm 0.30$ & $0.13 \pm 0.45$ & 0.52 & $83 \pm 29$ & \\
\hline 2006-10-12 04:19:47 & 8.10 & 2.66 & 1.71 & $R$ & & $-1.12 \pm 0.32$ & $-0.52 \pm 0.33$ & 1.23 & $102 \pm 10$ & \\
\hline 2006-10-12 04:40:12 & 8.11 & 2.66 & 1.71 & $r_{\text {cont }}$ & & $-0.95 \pm 0.49$ & $0.02 \pm 0.98$ & 0.95 & $89 \pm 30$ & \\
\hline 2006-10-12 04:56:35 & 8.12 & 2.66 & 1.71 & $R$ & $15.45 \pm 0.07$ & & & & & \\
\hline 2006-10-12 04:58:06 & 8.12 & 2.66 & 1.71 & V & $15.93 \pm 0.09$ & & & & & \\
\hline 2006-10-12 05:02:34 & 8.12 & 2.66 & 1.71 & $R$ & $15.45 \pm 0.07$ & & & & & [1] \\
\hline 2006-10-12 05:04:11 & 8.12 & 2.66 & 1.71 & $V$ & $15.98 \pm 0.08$ & & & & & [1] \\
\hline 2006-10-20 00:21:07 & 11.79 & 2.60 & 1.71 & $R$ & $15.68 \pm 0.07$ & & & & & \\
\hline 2006-10-20 00:22:28 & 11.79 & 2.60 & 1.71 & V & $16.12 \pm 0.07$ & & & & & [2] \\
\hline 2006-10-20 00:33:30 & 11.79 & 2.60 & 1.71 & V & & $0.07 \pm 0.42$ & $-0.36 \pm 0.42$ & 0.37 & $141 \pm 38$ & \\
\hline 2006-10-20 00:49:18 & 11.80 & 2.60 & 1.71 & $b_{\text {cont }}$ & & $-0.46 \pm 0.71$ & $0.43 \pm 0.66$ & 0.63 & $68 \pm 44$ & \\
\hline 2006-10-20 01:05:16 & 11.80 & 2.60 & 1.71 & $R$ & & $-0.24 \pm 0.32$ & $0.05 \pm 0.31$ & 0.24 & $85 \pm 43$ & \\
\hline 2006-10-20 01:25:05 & 11.81 & 2.60 & 1.71 & $r_{\text {cont }}$ & & $-1.54 \pm 0.82$ & $-0.01 \pm 0.67$ & 1.54 & $90 \pm 12$ & \\
\hline $2006-10-28$ 02:43:33 & 15.46 & 2.53 & 1.75 & $R$ & & $-0.31 \pm 0.15$ & $0.08 \pm 0.15$ & 0.32 & $83 \pm 17$ & \\
\hline 2006-10-28 03:18:40 & 15.47 & 2.53 & 1.75 & $V$ & & $0.37 \pm 0.20$ & $-0.30 \pm 0.19$ & 0.48 & $160 \pm 17$ & \\
\hline 2006-10-28 03:37:07 & 15.48 & 2.53 & 1.75 & $R$ & $15.85 \pm 0.07$ & & & & & \\
\hline 2006-10-28 03:38:38 & 15.48 & 2.53 & 1.75 & $V$ & $16.17 \pm 0.07$ & & & & & \\
\hline 2006-11-04 00:41:39 & 18.32 & 2.47 & 1.73 & $R$ & & $0.92 \pm 0.33$ & $-0.13 \pm 0.34$ & 0.93 & $176 \pm 12$ & \\
\hline 2006-11-04 00:59:12 & 18.32 & 2.47 & 1.73 & $V$ & & $0.89 \pm 0.43$ & $-0.30 \pm 0.43$ & 0.94 & $171 \pm 17$ & \\
\hline 2006-11-04 01:28:12 & 18.33 & 2.47 & 1.73 & $R$ & $15.97 \pm 0.14$ & & & & & \\
\hline 2006-11-04 01:29:45 & 18.33 & 2.47 & 1.73 & V & $16.40 \pm 0.22$ & & & & & \\
\hline 2006-11-07 00:39:12 & 19.47 & 2.44 & 1.74 & $R$ & & $0.56 \pm 0.21$ & $-0.14 \pm 0.19$ & 0.58 & $173 \pm 12$ & \\
\hline 2006-11-07 01:14:18 & 19.47 & 2.44 & 1.74 & $V$ & & $1.13 \pm 0.25$ & $-0.12 \pm 0.25$ & 1.13 & $177 \pm 7$ & \\
\hline 2006-11-07 01:34:45 & 19.47 & 2.44 & 1.74 & $R$ & $15.97 \pm 0.10$ & & & & & \\
\hline 2006-11-07 01:36:19 & 19.47 & 2.44 & 1.74 & V & $16.58 \pm 0.10$ & & & & & \\
\hline 2006-11-10 00:56:40 & 20.54 & 2.42 & 1.75 & $R$ & & $1.14 \pm 0.22$ & $-0.02 \pm 0.22$ & 1.14 & $179 \pm 6$ & \\
\hline 2006-11-10 01:32:37 & 20.55 & 2.42 & 1.75 & $V$ & & $0.55 \pm 0.25$ & $0.32 \pm 0.23$ & 0.64 & $15 \pm 15$ & \\
\hline 2006-11-10 01:53:11 & 20.55 & 2.42 & 1.75 & $R$ & $15.98 \pm 0.08$ & & & & & \\
\hline 2006-12-14 01:08:21 & 27.97 & 2.09 & 1.94 & $R$ & & $1.90 \pm 0.40$ & $-0.37 \pm 0.41$ & 1.93 & $175 \pm 7$ & [3] \\
\hline 2006-12-14 01:15:21 & 27.97 & 2.09 & 1.94 & $V$ & & $1.20 \pm 0.96$ & $-1.51 \pm 1.12$ & 1.93 & $154 \pm 22$ & [3] \\
\hline
\end{tabular}

Explanations: the table lists the observing epoch (midpoint of exposure series), the Sun $(r)$ and Earth $(\Delta)$ distances, the phase angle $(\phi)$, the filter used, the absolute filter brightness $m(1,1, \phi)$ for $r=\Delta=1$ AU and phase angle $\phi$, the Stokes parameters $P_{Q}$ and $P_{U}$, the fraction of linear polarization $P_{\mathrm{L}}$ and the angle $\zeta$ of maximum polarization. Comments are indexed and explained below: [1] photometric exposures in $V$ and $R$ were repeated because of guide probe vignetting; [2] no zeropoints available in $V$ on 2006-10-20. We used those obtained on 2006-10-19; [3] target very close to a bright star, accurate polarimetry and photometry impossible.

Linear polarization was then calculated from

$q^{\prime}=\frac{2}{N} \sum_{i=0}^{N} r\left(\alpha_{i}\right) \cos \left(4 \alpha_{i}\right)$,

and

$u^{\prime}=\frac{2}{N} \sum_{i=0}^{N} r\left(\alpha_{i}\right) \sin \left(4 \alpha_{i}\right)$,

where $q^{\prime}$ and $u^{\prime}$ are the reduced Stokes parameters defined according to Shurcliff (1962) and measured using as the reference direction the North Celestial Meridian, and $\alpha_{i}=22.5^{\circ} \times i$ are the position angles of the retarder waveplate. From $q^{\prime}$ and $u^{\prime}$, we finally calculated the linear polarization $P_{Q}=Q / I$ and $P_{U}=U / I$, by adopting as the reference direction the perpendicular to the great circle passing through the comet and the Sun at the observing epoch, as explained in Landi Degl'Innocenti et al. (2007). $P_{Q}$ and $P_{U}$ allow one to determine the total fraction of linear polarization $P_{\mathrm{L}}=\sqrt{P_{Q}^{2}+P_{U}^{2}}$ and the angle of maximum polarization $\zeta . P_{\mathrm{L}}$ represents the flux perpendicular to the plane Sun-Comet-Earth (the scattering plane) minus the flux parallel to that plane, divided by the sum of the two fluxes, and $\zeta$ is the angle between the perpendicular to the scattering plane and the direction of maximum polarization. As for the photometry, a fixed aperture of $15^{\prime \prime}$ diameter was applied for the polarimetric measurements of the comet. In the presence of coma, we have implicitly assumed that the polarization of the coma does not depend on $\rho$ (see below for a discussion of the influence of a faint coma on the polarization data of the comet).

\section{Results}

Search for coma: no coma is detectable by direct visual inspection of the comet images. To confirm the presence of a faint coma possibly undetectable by visual inspection and measure its contribution to the object brightness, two numerical analysis 


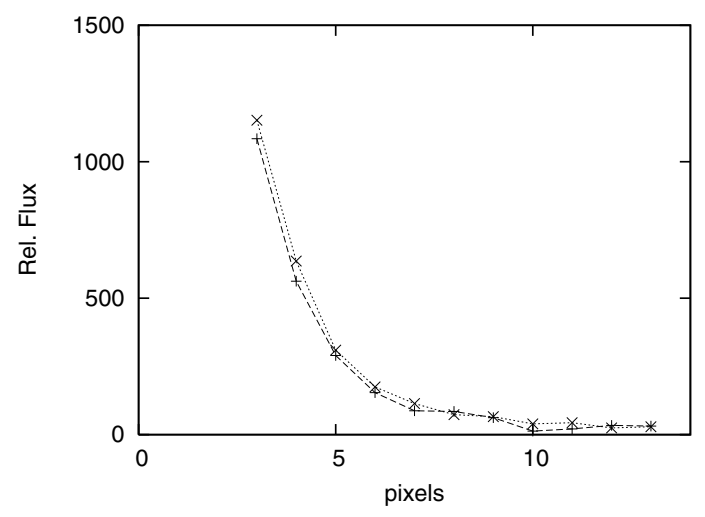

Fig. 1. Azimuthally averaged PSF profile of the comet (dashed line) compared with that of a background star in the same exposure. The image was taken on 28 Oct. 2006 through the $V$ filter.

methods were applied. The first and classical method (see for instance Boehnhardt et al. 2002) compared the azimuthally averaged profiles of the comet with the instrumental point spread function (PSF) measured from background stars in the same exposure. Since the comet had a proper motion and the telescope was tracking the comet, the stars appeared as short trails. Hence, the stellar PSF can only be measured in one direction i.e. perpendicular to the cometary motion and averaged along the trail direction. Figure 1 shows that the stellar and cometary PSF are almost identical, in particular in the PSF wings and close to the background level at which the coma light is expected to provide the most significant contribution.

To increase the sensitivity to coma detection, all polarimetric images recorded in a single filter during a single observing night were centered on the comet position and coadded, reaching a total integration time of approximately $1000 \mathrm{~s}$ (instead of the short $60 \mathrm{~s}$ exposures used for normal photometric observations). Due to seeing variations during exposure series, direct comparison of the PSFs of comet and background stars was complicated and usually impossible. For these composite images a second analysis method was therefore applied: measurement of the $\Sigma A f$ as function of $\rho$ (see above). In the case of the absence of coma, this function should approach zero at radial distance $\rho$ of about $3 F W H M$ from the central brightness peak, with $F W H M$ being the value of the full width at half maximum of the PSF of the coadded comet image. In the case of the coadded polarimetric images of $2 \mathrm{P} /$ Encke, all $\Sigma A f$ profiles show small, but significant non-zero fluxes at larger distances from the center of the coadded comet image. We recall that the background level has been subtracted using $\Sigma A f$ as described above and that any residual background level would result in a (likely linear) non-constant trend of $\Sigma A f$ versus $\rho$. The fact that for the $2 \mathrm{P} /$ Encke polarimetric images $\Sigma A f$ is constant with $\rho$ means that the intensity profile of the comet declines with $1 / \rho$, a phenomenon that is typical of an expanding dust coma around the nucleus. We therefore conclude that a weak coma was present around the comet during the entire period of our observations, i.e. from early Oct. to mid Dec. 2006 when the comet was approaching the Sun from 2.75 to $2.1 \mathrm{AU}$. Given the overall $\Sigma A f$ profile, it is unlikely that the central brightness peak of the comet images is due to an unresolved dense dust coma; instead, we consider the photometric flux of the peak produced by surface-reflected sunlight.

Flux calibration of the polarimetric images was obtained by comparing the inner part of the $\Sigma A f$ profile of the coadded polarimetric image with that of a calibrated normal filter

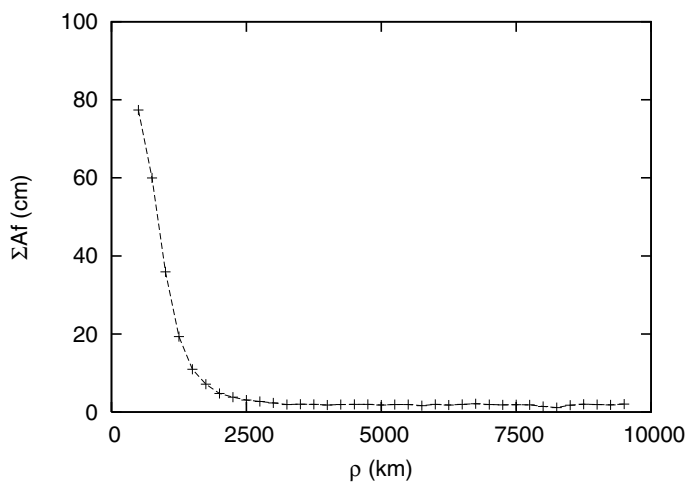

Fig. 2. $\Sigma A f$ profile as function of the nucleocentric distance $\rho$, obtained from polarimetric observations in $V$ filter on 28 Oct. 2008. The total exposure time is $960 \mathrm{~s}$. For radial distances greater than $2000 \mathrm{~km}, \Sigma A f$ is small but different from the background level of zero flux. Hence a very faint coma is present around the cometary nucleus.

exposure of the comet taken in the same filter during the same night. Figure 2 shows an example of the $\Sigma A f$ profile of a coadded polarimetric image series of comet $2 \mathrm{P} /$ Encke. Within the error margins, the $\Sigma A f$ profiles obtained for the various observing nights show no trend, neither with heliocentric distance nor with phase angle. The average level of the weak coma flux corresponds to $A f \rho$ values (for the definition of $A f \rho$ see above) of $0.65 \pm 0.35 \mathrm{~cm}$ in $V$ and $0.49 \pm 0.19 \mathrm{~cm}$ in $R$. They are equivalent to a dust production rate $Q_{\text {dust }}$ of the order of about $0.05 \mathrm{~kg} / \mathrm{s}$, assuming a simple empirical relationship between $A f \rho$ and $Q_{\text {dust }}$ used by Kidger (2004). The measured $A f \rho$ values equal no more than 1 percent of the total cometary signal and its effect on the photometric and polarimetric data analysis of $2 \mathrm{P} /$ Encke described below, can be neglected. We also note that during the reduction of the polarization images a weak extended coma was subtracted as part of the general background signal measured beyond $3 F W H M$ of the PSF of the comet. Only second order contributions to the $Q$ and $U$ measurements of the comet signal may remain from polarized light of the dust coma around the nucleus. Since estimated to be below 1 percent of the measured Stokes parameters, we ignored these in the overall error analysis of the polarimetric results of $2 \mathrm{P} /$ Encke.

Photometric phase function of the nucleus: Fig. 3 shows the photometric phase function of the nucleus of comet $2 \mathrm{P} /$ Encke for broadband $V$ and $R$ filters. The values plotted therein, the so-called $m(1,1, \phi)$ magnitudes (see Table 1$)$, are derived from the observed filter magnitudes by removing the dependencies on the Sun and Earth distances for the respective observing epochs. The figure shows a steep almost linear brightness increase for the nucleus with decreasing phase angle. It also suggests that there is - at least in the $R$ filter data - a slight nonlinear increase in brightness toward zero phase, starting all the way from $\phi=20^{\circ}$. Although this deviation from linear slope is within the expected amplitude of brightness variations due to the rotation of the non-spherical nucleus, its systematics suggest that the opposition effect may play a role in the nucleus phase function of $2 \mathrm{P} /$ Encke. Ignoring the possible minor nonlinearity in the phase functions of the comet, we found almost identical slopes $\beta$ when fitting a linear phase curve, i.e. for the $V$ filter data $\beta=0.051 \pm 0.004 \mathrm{mag} /{ }^{\circ}$ and for the $R$ filter ones $\beta=0.049 \pm 0.004 \mathrm{mag} /{ }^{\circ}$. Our $\beta$ is slightly smaller, although consistent within the error margins, than the linear slope parameter ( $\left.\beta=0.06 \mathrm{mag} /{ }^{\circ}\right)$ determined by Fernandez et al. (2000) from a compilation of published nucleus magnitudes of the comet 


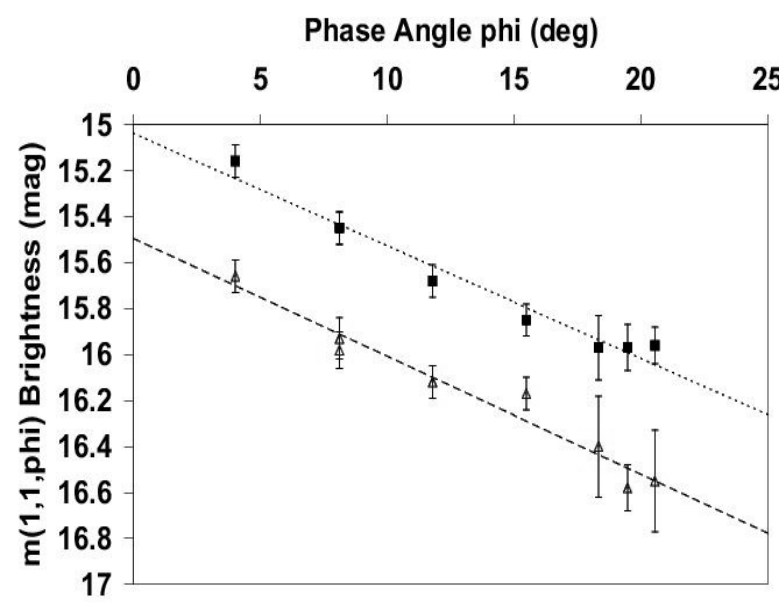

Fig. 3. The brightness phase function of the nucleus of comet 2P/Encke in broadband $V$ and $R$ filters. The plot shows the measured $m(1,1, \phi)$ brightness, in addition to the linear fit functions wersus phase angle. Filled squares $=R$ filter, open triangles $=V$ filter.

measured over a wider phase-angle range and that found by Jewitt (2004) for $2 \mathrm{P} /$ Encke $\left(\beta=0.060 \pm 0.005 \mathrm{mag} /{ }^{\circ}\right)$ derived from a much sparser dataset. We conclude that the phase function of $2 \mathrm{P} /$ Encke follows a color-neutral almost linear brightening law with $\beta=0.050 \pm 0.004 \mathrm{mag} /{ }^{\circ}$, which is slightly steeper than the canonical value $\left(\beta=0.04 \mathrm{mag} /{ }^{\circ}\right.$; Lamy et al. 2004), although within the range found for other cometary nuclei (Jewitt 2004; Lamy et al. 2004).

Nucleus size and colors: extrapolation of the linear phase function of $2 \mathrm{P} /$ Encke to zero phase angle provides the absolute magnitude $m(1,1,0)$ of the nucleus, i.e. $15.50 \pm 0.06$ and $15.03 \pm 0.05 \mathrm{mag}$ for $V$ and $R$ filter, respectively. With the geometric albedo of 0.047 (Lamy et al. 2004; Fernandez et al. 2000), we obtain an equivalent radius of $2.43 \pm 0.06 \mathrm{~km}$ in $R$, in good agreement with the radius estimate by Fernandez et al. (2000; $2.4 \pm 0.3 \mathrm{~km}$ ), Kelley et al. (2006), and Campins et al. (1988) and slightly smaller than radar echo results by Harmon \& Nolan $(2005 ; 2.42-3.72 \mathrm{~km})$. We emphasize that our results may be affected by insufficient sampling of the rotation light curve and may deviate from earlier findings because of different viewing aspects of the nucleus along the orbit and with time (see Belton et al. 2005). The $V-R$ color of the nucleus is $0.47 \pm 0.07 \mathrm{mag}$, which corresponds to an intrinsic color (i.e. corrected for solar $V-R$ color) of $0.11 \pm 0.07 \mathrm{mag}$ or a spectral slope $S^{\prime}$ of $11 \pm$ $8 \% / 100 \mathrm{~nm}$. Our mean $V-R$ color and the spectral gradient $S^{\prime}$ are slightly higher than that measured by Jewitt (2004) and Lowry \& Weissman (2007), although still within the error margins.

Polarimetric phase function of the nucleus: Stokes $P_{Q}$ and $P_{U}$ of the linear polarimetry measured for $2 \mathrm{P} /$ Encke are compiled in Table 1 . The $P_{Q}$ and $P_{U}$ values listed refer to the scattering plane Sun-comet-observer. The polarization $P_{L}$ with respect to the scattering plane (listed in Table 1) is plotted versus phase angle in Fig. 4 for the two broadband filters used ( $V$ and $R$ ). In both broadband filters the polarization shows the same, linear increase with increasing phase angle $\phi$ (from about $-1 \%$ at $\phi=$ $4^{\circ}$ to about $+1.7 \%$ at $\phi=28^{\circ}$ ). The minimum polarization values for $V$ and $R$ correspond to the upper limits only, since our polarization phase functions do not show the expected turn-over towards zero polarization for small phase angles (the minimum polarization is rather the lower end of the linear phase function slope and the turn-over occurs at $\phi<4^{\circ}$ only). Zero polarization is passed at about $13^{\circ}$ phase angle (inversion angle). The slope of

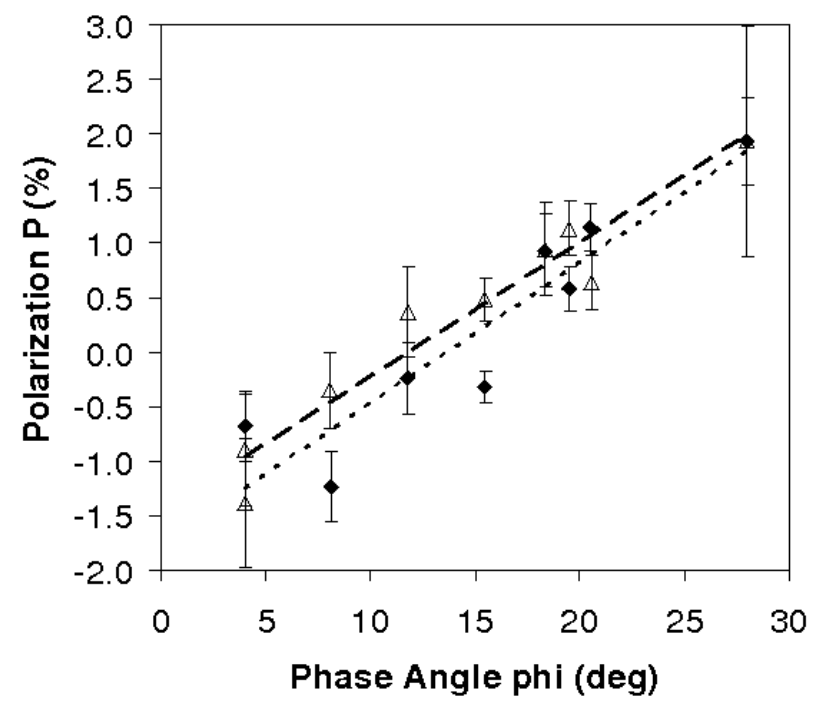

Fig. 4. Linear polarization $P$ of comet $2 \mathrm{P} /$ Encke versus phase angle. Filled triangles/squares $=V / R$ filter measurements, long/short dash lines = linear fits to $V / R$ filter measurements.

the polarization increase is determined by linear regression to be $0.123 \pm 0.013 \% /{ }^{\circ}$ in $V$ and $0.129 \pm 0.021 \% /{ }^{\circ}$ in $R$, i.e. within the errors the phase-angle gradients are identical and do not change with filter.

From the values listed in Table 1, it is obvious that for small $P_{Q}$, i.e. close to the overall measurement uncertainty, Stokes $P_{U}$ has comparable amplitudes to $P_{Q}$ which produces a considerable scatter and large uncertainties in the values for the position angle $\zeta$ of maximum linear polarization. The position angle $\zeta$ is well determined for higher polarization values and agrees well with the position angle of the light scattering plane. However, the close-to-zero level of Stokes $P_{U}$ for all measurements provides evidence for the correctness of the data reduction, and is in agreement with theoretical expectations for a light-scatteringplane oriented polarization (see Muinonen et al. 2002). For the narrowband measurements using filters $b_{\text {cont }}$ and $r_{\text {cont }}$, Stokes $P_{Q}$ is systematically higher for the red continuum filter compared with the blue, although all results are affected by relatively large errors. Within the margins of the errors the measured Stokes parameters agree with the Stokes parameters measured for the $V$ and $R$ filters, respectively.

To our knowledge, $2 \mathrm{P} /$ Encke is the only comet that has linear polarization at the nucleus measured. Jewitt (2004) published a set of polarization values $(1.5,1.0,1.9 \pm 0.5 \%)$, obtained on 22 Oct. 2003 at phase angle $22.1^{\circ}$ through cometary dust continuum filters (central wavelengths of 445.3, 525.9, $713.3 \mathrm{~nm}$ ). His results are consistent with our own.

Test of the asteroid polarization-albedo relationship: we also applied the polarization-albedo relationship for asteroids (Zellner \& Gradie 1976; Zellner et al. 1977) to the 2P/Encke polarimetry data to validate this empirical method of albedo estimation for the nucleus of the comet. Using the phase function parameters of Lupishko \& Mohamed (1996) we derive a geometric albedo of 0.145 (range $0.13-0.16$ ), when using the average slope of the polarization phase function (see above), and of 0.08 (range 0.06-0.16) when using the values of minimum polarization $P_{\min }-$ which by itself is an upper limit only. Unfortunately, in our measurements of $2 \mathrm{P} /$ Encke $P_{\min }$ is not well determined and the upper limit used instead shows relatively large errors (see Table 1). In conclusion, the empirical polarizationalbedo relationships for asteroids suggests a significantly higher 
Table 2. Comparison of photometric and polarimetric properties of comet Encke and other small bodies.

\begin{tabular}{|c|c|c|c|c|c|c|c|c|}
\hline 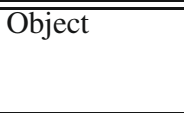 & $\begin{array}{c}\text { Albedo } \\
p_{V}\end{array}$ & $\begin{array}{c}\text { Phase coefficient, } \\
\beta \text { for } 5 \leq \phi \leq 30^{\circ} \\
\left(\mathrm{mag} /{ }^{\circ}\right)\end{array}$ & $\begin{array}{l}\text { Spectral } \\
\text { slope } \\
(\% / 100 \mathrm{~nm})\end{array}$ & $\begin{array}{c}P_{\min } \mid \\
(\%)\end{array}$ & $\begin{array}{c}\phi_{\min } \\
\left({ }^{\circ}\right)\end{array}$ & $\begin{array}{c}\text { Pol. slope } \\
\left(\% /^{\circ}\right)\end{array}$ & $\begin{array}{c}\text { Pol. Inv. } \\
\text { angle } \phi_{o} \\
\left({ }^{\circ}\right)\end{array}$ & $\begin{array}{l}\text { Pol. spect. } \\
\text { gradient } \\
\text { at } \phi \leq 30^{\circ}\end{array}$ \\
\hline 2P/Encke & 0.047 & 0.048 & 11 & $\geq 1.4$ & $\leq 4$ & 0.123 & 13 & $\begin{array}{l}\text { negative } \\
\text { decreases with } \phi\end{array}$ \\
\hline Comet nuclei & $0.02-0.06$ & $0.043-0.059$ & $0-41$ & $?$ & $?$ & $?$ & $?$ & $?$ \\
\hline Comet dust & 0.05 & $0.018-0.035$ & $-1-12$ & 1.5 & 9.6 & 0.31 & 21.8 & \multirow{2}{*}{$\begin{array}{l}\text { negative } \\
\text { decreases with } \phi \\
\text { negative? }\end{array}$} \\
\hline 2060/Chiron & 0.07 & 0.045 & 1 & 1.5 & $1.5-2$ & Out of range & $9(?)$ & \\
\hline $\mathrm{C}$ asteroids & $0.02-0.09$ & 0.042 & $-3-20$ & 1.73 & 9.4 & 0.28 & 20.5 & \multirow{3}{*}{$\begin{array}{l}\text { positive } \\
\text { increases with } \phi \\
\text { negative } \\
\text { increases with } \phi\end{array}$} \\
\hline $\mathrm{S}$ asteroids & $0.085-0.33$ & 0.029 & $-15-88$ & 0.77 & 6.3 & 0.09 & 20.1 & \\
\hline E asteroids & $0.34-0.6$ & 0.019 & rather flat spectra & 0.3 & 5.8 & 0.04 & 17.8 & \\
\hline M asteroids & $0.074-0.25$ & 0.033 & $6-15$ & 0.96 & 7.7 & 0.09 & 23.5 & \multirow{2}{*}{$\begin{array}{l}\text { negative } \\
\text { increases with } \phi \\
\text { slightly negative or } \\
\text { neutral }\end{array}$} \\
\hline $\mathrm{F}$ asteroids & $0.024-0.086$ & 0.044 & $\begin{array}{c}-24 \text { (Interamnia) } \\
\text { bluish spectra }\end{array}$ & $1-1.4$ & $7.6-8$ & $0.35-0.4$ & $14-17$ & \\
\hline
\end{tabular}

Explanations: column "Spectral slope" lists the slope of the visible spectrum of the objects as determined from measured filter colors corrected for the color of the Sun (see Boehnhardt et al. 2001); " $\left|P_{\min }\right|$ " gives the value of minimum polarization, " $\phi_{\min }$ " the phase angle of $\left|P_{\min }\right|$, "Pol. slope" the gradient of the polarimetric phase function and "Pol. Inv." the polarization inversion angle, i.e. when the sign of polarization $P_{\mathrm{L}}$ changes from negative to positive. Column "Pol. spect. gradient at $\phi \leq 30^{\circ}$ " describes the overall spectral shape of the object spectra for phase angles below $30^{\circ}$. The references for the table values are: albedo for comet nuclei - Lamy et al. (2004); filter colors of objects - Snodgrass et al. (2008); phase coefficients from space missions - Soderblom et al. (2002); Li et al. (2006) phase coefficients from ground-based observations - Jewitt \& Sheppard (2004); Jewitt et al. (2003); albedo for 2060/Chiron - Groussin et al. (2004); $V-R$ color of 2060/Chiron - Davies et al. (1998); the rest of 2060/Chiron's data - Bagnulo et al. (2006); asteroid albedos - Tedesco et al. (1989); asteroid phase coefficients - Bowell et al. (1979); asteroid filter colors - Hansen (1976); Chapman \& Gaffey (1979); polarimetric asteroid data - Lupishko (2006); spectral gradients of polarization for asteroids - Belskaya et al. (2008); comet dust data - Kolokolova et al. (2004); Kiselev \& Rosenbush (2004); Meech \& Jewitt (1987). Question marks indicate that no comparison data are available.

geometric albedo for the nucleus of $2 \mathrm{P} /$ Encke compared to that obtained from the more realistic approach of visible and thermal IR measurements of the nucleus (0.047; Lamy et al. 2004; Fernandez et al. 2000). This may indicate that either the empirical polarization-albedo relationship of asteroids does not apply to cometary nuclei, or at least not for $2 \mathrm{P} /$ Encke, or, if applicable, that it requires different fitting parameters than determined from asteroids. It is indeed been proposed that the empirircal polarization-albedo rule is not applicable to extremely dark objects such as cometary nuclei (Dollfus \& Zellner 1979). The negative outcome of this test may imply that the surface constitution of 2P/Encke differs from those of main-belt and near-Earth asteroids for which the empirical relationship is calibrated by measurements.

\section{Discussion and conclusions}

We have presented photometric and linear polarimetric phase functions of 2P/Encke measured during the 2006 approach of the comet to the Sun. The weak coma found about the comet does not affect significantly the photometric and polarimetric light-scattering phase functions measured, i.e. the phase functions reflect the light-scattering behavior of the nucleus surface. Both phase functions show an almost linear behavior with phase angle $\phi$ (over the measured range from 4 to $28 \mathrm{deg}$ ). Trends in the phase function with wavelength are not obvious for the nucleus photometry, but may exist for the linear polarization. A small opposition surge is tentatively suggested in the photometric data, but needs verification by further observations at smaller phase angles. Similarly, the value of minimum polarization $P_{\min }$ in the polarization phase curve should be reassessed by new observations covering the range to zero phase angle that is of immediate interest for modeling the opposition effects in the lightscattering by the cometary nucleus. Our radius estimation and spectral slope $S^{\prime}$ agree with earlier measurements, in particularly well with those obtained from IR observations. The low activity level of the nucleus at solar distances of 2.75 to $2.1 \mathrm{AU}$ suggests that the cometary nucleus is covered by a crust that prevents major outgassing. This appears to be true during this part of the orbit, even though $2 \mathrm{P} /$ Encke has entered the distance range where water sublimation usually generates significant cometary activity.

Table 2 compares light-scattering properties of the nucleus of comet 2P/Encke with those of other solar-system bodies, that may have similar surfaces and were observed in similar conditions (phase angle, wavelength). The majority of the data are for $V$ filter - if not indicated otherwise in the table at the phase angles $5-30^{\circ}$. The analysis of Table 2 indicates that comet $2 \mathrm{P} /$ Encke displays photometric properties typical of cometary nuclei. As for other cometary nuclei, the slope of the phase function differs from that measured for cometary dust, which might be due to the difference between light scattering on individual dust particles and their compact layers at the nuclear surfaces.

Among asteroids, the light scattering behavior of C-type objects resembles most closely that of comet $2 \mathrm{P} /$ Encke, at least its photometric properties. There are major differences between the polarization phase curve (Fig. 4 ) of the nucleus of $2 \mathrm{P} /$ Encke and 
the polarization phase curves of asteroids of different taxonomy. Primitive asteroids of type C, P, G, and B and evolved asteroids of type $\mathrm{V}, \mathrm{S}, \mathrm{M}, \mathrm{E}$, and $\mathrm{A}$ have, in general, larger inversion angles than 2P/Encke (cf. Muinonen et al. 2002; Fornasier et al. 2006), while only primitive F-type asteroids exhibit similar inversion angles (Belskaya et al. 2005). However, other polarimetric characteristics of F-type asteroids differ significantly: the angle of the polarization minimum is at least twice as large, and the slope of polarization at the inversion angle is almost three times larger than that of comet 2P/Encke. Moreover, F-type asteroids do not exhibit red colors, but display neutral or even bluish surface colors. Unfortunately, we cannot compare the properties of comet 2P/Encke with icy bodies such as Transneptunian objects or the small satellites of Saturn, Uranus, and Neptune since these objects can be observed only at small phase angles. The Centaur 2060/Chiron may be the object most appropriate for the comparison. Compared to comet $2 \mathrm{P} /$ Encke, it has however neither similar photometric (too high albedo, too red color) nor polarimetric data (too small inversion angle). The polarimetry therefore reveals unique properties of the nucleus of comet $2 \mathrm{P} /$ Encke that may be typical for cometary nuclei but differ significantly from the properties of other solar-system bodies. This uniqueness does not manifest itself photometrically. It may indicate a specific composition or structure of the surface layer of cometary nuclei. Whether the narrow negative polarization branch can be explained qualitatively by the single-scattering interference mechanism (Muinonen et al. 2007) remains to be answered by a future study. Based on that mechanism, negative polarization branches of icy objects which corresponds to smaller real parts of refractive indices, can be expected to be narrower than those of silicate-rich stony objects, which represents larger real parts of refractive indices.

The polarization minimum of $2 \mathrm{P} /$ Encke, which is not well constrained by the present data set, is less than or equal to $-1 \%$ and, as mentioned above, it is possibly within the range of data for F-type asteroids that have polarization minima between about -1.0 and $-1.5 \%$ (Belskaya et al. 2005). However, we reiterate that the polarization phase curve of $2 \mathrm{P} /$ Encke is the only data set for comets available to ourselves. The number of F-type asteroids with measured polarization phase curves is also small such that, in terms of polarization properties, it is difficult to decide whether comets and F-type asteroids are similar.

Our 2P/Encke results have shown that the empirical albedopolarization relationships of asteroids cannot easily be applied to cometary nuclei, possibly because the surface constitution and light-scattering properties are different. Whether such a relationship can be established for cometary nuclei remains undecided until more objects are studied and a self-consistent modeling of photometric, spectroscopic, and polarimetric light-scattering properties for comets is available.

Acknowledgements. We would like to thank everyone at the European Southern Observatory at Cerro Paranal in Chile and in Garching/Germany, who were involved in the implementation and execution of this service mode program at the Very Large Telescope VLT.

\section{References}

A'Hearn, M. F., Millis, R. L., Schleicher, D. G., et al. 1995, Icarus, 118, 223 Bagnulo, S., Boehnhardt, H., Muinonen, K., et al. 2006, A\&A, 450, 1239 Belskaya, I. N., Skhuratov, Y., Efimov, Y., et al. 2006, Icarus, 178, 213

Belskaya, I., Levasseur-Regourd, A.-C., Cellino, A., et al. 2008, Icarus, in press Belton, M. J. S., Samarasinha, N. H., Fernandez, Y. R., \& Meech, K. J. 2005, Icarus, 175, 181

Boehnhardt, H., Tozzi, G. P., Birkle, K., et al. 2001, A\&A, 378, 653 Boehnhardt, H., Delahodde, C., Sekiguchi, T., et al. 2002, A\&A, 387, 1107 Boehnhardt, H., Bagnulo, S., Muinonen, K., et al. 2004, A\&A, 415, L21 Bowell, E., Gehrels, T., \& Zellner, B. 1979, in Asteroids, ed. T. Gehrels (Tucson: Univ. Arizona Press), 1108

Campins, H. 1988, Icarus, 73, 508

Cellino, A., Gil Hutton, R., Tedesco, E. F., et al. 1999, Icarus, 138, 129

Chapman, C. R., \& Gaffey, M. J. 1979, in Asteroids, ed. T. Gehrels (Tucson: Univ. Arizona Press), 1064

Davies, J. K., McBride, N., Ellison, S. L., et al. 1998, Icarus, 134, 213

Dollfus, A., \& Zellner, B. 1979, in Asteroids, ed. T. Gehrels (Tucson: Univ. Arizona Press), 170

Fernandez, Y. R., Lisse, C. M., Käufl, H. U., et al. 2000, Icarus, 147, 145 Fernandez, Y. R., Lowry, S. C., Weissman, P. R., et al. 2005, Icarus, 175, 194 Festou, M. C., \& Barale, O. 2000, AJ, 119, 3119

Fornasier, S., Belskaya, I. N., Shkuratov, Y., et al. 2006, A\&A, 455, 371

Groussin, O., Lamy, P., \& Jorda, L. 2004, A\&A, 413, 1163

Hansen, O. L. 1976, AJ, 81, 74

Harmon, J. K., \& Nolan, M. C. 2005, Icarus, 176, 175

Jewitt, D. 2002, AJ, 123, 1039

Jewitt, D. 2004, AJ, 128, 3061

Jewitt, D., \& Sheppard, S. 2004, AJ, 127, 1784

Jewitt, D., Sheppard, S., \& Fernández, Y. 2003, AJ, 125, 3366

Kelley, M. S., Woodward, C. E., Harker, D. E., et al. 2006, AJ, 651, 1256

Kidger, M. 2004, A\&A, 420, 389

Kiselev, N., \& Rosenbush, V. 2004, in Photopolarimetry in Remote Sensing, ed. G. Videen, Ya. Yatskiv, \& M. Mishchenko (Dordrecht: Kluwer Academic Publishers), 411

Kolokolova, L., Hanner, M., Levasseur-Regourd, A.-C., \& Gustafson, B. A. S. 2004, in Comets II, ed. M. C. Festou, H. U. Keller, \& H. A. Weaver (Tucson: Univ. Arizona Press), 577

Lamy, P. L., Toth, I., Fernandez, Y. R., \& Weaver, H. A. 2004, in Comets II, ed. M. C. Festou, H. U. Keller, \& H. A. Weaver (Tucson: Univ. Arizona Press), 223

Landi Degl'Innocenti, E., Bagnulo, S., \& Fossati, L. 2007, in The future of photometric, spectrophotometric, and polarimetric standardization, ed. C. Sterken, ASP Conf. Ser., 364, 495

Levison, H. F., Terrell, D., Wiegert, P. A., et al. 2006, Icarus, 182, 161

Li, J. Y., A'Hearn, M. F., Belton, M. J. S., et al. 2007, Icarus, 191, 161

Lowry, S. C., \& Weissman, P. R. 2007, Icarus, 188, 212

Lupishko, D. F. 2006, Asteroid Polarimetric Database V5.0., EAR-A-3-RDRAPD-POLARIMETRY-V5.0. NASA Planetary Data System

Lupishko, D. F., \& Mohamed, R. A. 1996, Icarus, 11, 209

Meech, K. J., \& Jewitt, D. C. 1987, A\&A, 187, 585

Muinonen, K., Piironen, J., Shkuratov, Y., et al. 2002, in Asteroids III, ed. W. F. Bottke, A. Cellino, P. Paolicchi, \& R. P. Binzel (Tucson: Univ. Arizona Press), 123

Muinonen, K., Zubko, E., Tyynelä, J., et al. 2007, J. Quant. Spectrosc. Radiat. Trans., 106, 360

Sekanina, Z. 1991, in Comets in the Post-Halley Era, ed. R. L. Newburn, M. Neugebauer, \& J. Rahe (Dordrecht: Kluwer Press), 769

Shurcliff, W. A. 1962, Polarized light (Cambridge: Harward University Press) Snodgrass, C., Lowry, S. C., \& Fitzsimmons, A. 2008, MNRAS, 385, 737 Soderblom, L. A., Becker, T. L., Bennett, G., et al. 2002, Science, 296, 1087 Tedesco, E. F., Williams, J. G., Matson, D. L., et al. 1989, AJ, 97, 580 Tozzi, G. P., Lara, L. M., Kolokolova, L., et al. 2004, A\&A, 424, 325 Zellner, B., \& Gradie, J. 1976, AJ, 81, 262

Zellner, B., Leake, M., Bertre, T., et al. 1977, LPSC Conf. Ser. (Oxford: Pergamon Press), 1091 